\title{
Myocardial imaging using ultrahigh- resolution spectral domain optical coherence tomography
}

Xinwen Yao

Yu Gan

Charles C. Marboe

Christine P. Hendon 


\title{
Myocardial imaging using ultrahigh-resolution spectral domain optical coherence tomography
}

\author{
Xinwen Yao, ${ }^{a}$ Yu Gan, ${ }^{a}$ Charles C. Marboe, ${ }^{b}$ and Christine P. Hendon ${ }^{a, *}$ \\ ${ }^{a}$ Columbia University, Department of Electrical Engineering, 500 West 120th Street, New York, New York 10027, United States \\ ${ }^{b}$ Columbia University Medical Center, Department of Pathology and Cell Biology, 630 West 168th Street, New York, New York 10032, United States
}

\begin{abstract}
We present an ultrahigh-resolution spectral domain optical coherence tomography (OCT) system in $800 \mathrm{~nm}$ with a low-noise supercontinuum source (SC) optimized for myocardial imaging. The system was demonstrated to have an axial resolution of $2.72 \mu \mathrm{m}$ with a large imaging depth of $1.78 \mathrm{~mm}$ and a $6-\mathrm{dB}$ falloff range of $0.89 \mathrm{~mm}$. The lateral resolution $(5.52 \mu \mathrm{m})$ was compromised to enhance the image penetration required for myocardial imaging. The noise of the SC source was analyzed extensively and an imaging protocol was proposed for SC-based OCT imaging with appreciable contrast. Three-dimensional datasets were acquired ex vivo on the endocardium side of tissue specimens from different chambers of fresh human and swine hearts. With the increased resolution and contrast, features such as elastic fibers, Purkinje fibers, and collagen fiber bundles were observed. The correlation between the structural information revealed in the OCT images and tissue pathology was discussed as well. ๑ The Authors. Published by SPIE under a Creative Commons Attribution 3.0 Unported License. Distribution or reproduction of this work in whole or in part requires full attribution of the original publication, including its DOI. [DOI: 10.1117/1.JBO.21.6.061006]
\end{abstract}

Keywords: optical coherence tomography; supercontinuum source; biomedical imaging; myocardial imaging; cardiology.

Paper 150716SSRR received Oct. 26, 2015; accepted for publication Feb. 29, 2016; published online Mar. $22,2016$.

\section{Introduction}

Cardiac diseases are among the leading causes of morbidity and mortality globally. During the progression of heart disease, microstructural heterogeneity within myocardial tissue could lead to an increased risk of life-threatening cardiac diseases such as ventricular arrhythmias and heart failure., ${ }^{1,2}$ Visualization of the features in the endomyocardial layer may shed light on the correlations between the microstructural and functional changes in the tissue, which are important for guiding diagnostic procedures and clinical treatment such as reverse remodeling therapy, ${ }^{3}$ radiofrequency ablation, ${ }^{4}$ and endomyocardial biopsy (EMB)., ${ }^{5,6}$ Particularly, despite the controversy of EMB, it is still the most frequently used method for surveillance of cardiac allograft rejection and for the diagnosis of unexplained ventricular dysfunction. ${ }^{6}$ The main reason is that most of the well-established medical imaging modalities (magnetic resonance imaging, ${ }^{7}$ echocardiography, ${ }^{8}$ and computed tomography ${ }^{9}$ ) do not have sufficient resolution to provide the cellular-level assessment of the myocardium that the biopsy can otherwise provide. ${ }^{5}$ Optical coherence tomography (OCT) is a high-speed, nondestructive imaging tool that is able to produce cross-sectional and three-dimensional (3-D) images with cellular/subcellular axial resolution within millimeter-scale penetration depth of biological samples and has been applied in a wide range of medical imaging applications. ${ }^{10,11}$

Previous works have demonstrated the feasibility of OCT to extract important information in the myocardium, including fiber orientation and identification of radiofrequency ablation lesions. ${ }^{12-14}$ These works were conducted in the $1300-\mathrm{nm}$ optical window mainly for higher penetration depth in the myocardium, while many details were still buried in the OCT

*Address all correspondence to: Christine P. Hendon, E-mail: cpf2115@ columbia.edu images. This may be due to the insufficient axial resolution as well as the relatively weak backscattering signal collected from the myocardium sample at this optical window. In order to characterize the pathology-related tissue types in the myocardium, higher resolution and better contrast of the OCT images are needed and can be achieved by moving to a shorter wavelength regime. Since OCT signals are generated based on the detection of backscattered light, another benefit to working in the shorter wavelength regime is that it favors stronger light scattering, which will provide more information about the structures inside the tissue. Moreover, the optical properties of different types of tissue in the shorter NIR regime are more diversified compared with the 1300-nm window. Working in this regime will enable functional extensions of the standard OCT imaging, such as spectroscopic OCT. Based on these reasons, most of the ultrahigh-resolution (UHR) works in OCT chose to employ light sources that were in the shorter NIR regime. ${ }^{15-24}$ In general, UHR OCT categorizes OCT systems with axial resolution lower than $5 \mu \mathrm{m}$ in air. The early UHR OCT systems were built in time domain (TD) configuration with the help of state-of-the-art femtosecond lasers to generate ultrashort pulses. ${ }^{15,16,25}$ After balancing the dispersion mismatch in the interference arms, an axial resolution as high as $0.75 \mu \mathrm{m}$ in air was reported. ${ }^{15}$ The high-lateral resolutions of these systems were achieved by adopting high-NA objectives in the sample arm, which was necessarily associated with a reduced depth of focus and eventually limited the imaging depth to around $0.5 \mathrm{~mm}$. Later on, UHR OCT was implemented in spectral domain (SD) configurations in both bench-top systems ${ }^{17-21}$ and endoscopic systems. ${ }^{22,23}$ SD-OCT is known to have a sensitivity advantage over its TD counterparts owing to the paralleled detection in the $\mathrm{SD},{ }^{24,26}$ and is more preferable in practice. The key component of UHR SD-OCT is the spectrometer. In particular, due to the finite sampling area of a single detector pixel, fringe visibility was compromised at higher spatial frequency, which causes a sensitivity falloff at deeper imaging 
ranges. For example, Liu et al. ${ }^{19}$ demonstrated an axial resolution of $1 \mu \mathrm{m}$ in air in the 800-nm spectral window using a spectrometer with a spectral range of around $400 \mathrm{~nm}$ captured by a 2-k pixel line camera. Despite the superb axial resolution, the imaging depth was limited to $0.5 \mathrm{~mm}$, mainly due to a low spectral resolution in the $k$-domain. Alternatively, Yadav et al. ${ }^{20}$ reported a UHR OCT system with $1.5-\mu \mathrm{m}$ axial resolution and $25-\mu \mathrm{m}$ lateral resolution in air. A modified CzernyTurner spectrometer was designed for a similar spectral range but with a detector of $8 \mathrm{k}$ pixels. Though not all of the pixels were used to cover the bandwidth, it helped to extend the imaging depth to around $1.2 \mathrm{~mm}$. However, the pixel size of $5 \mu \mathrm{m}$ led to an insufficient coupling efficiency and resulted in a lower SNR. Moreover, the limited spectral resolution as well as the inaccurate $k$-domain resampling method for data interpolation led to a 6-dB falloff range around $0.5 \mathrm{~mm}$. Recently, Xi et al. $^{23}$ reported a UHR SD-OCT endoscope with $3-\mu \mathrm{m}$ axial resolution in air produced by a linear- $k$ spectrometer, in which a more complicated design and alignment of the optics were required to achieve a good performance. Still, the reported imaging depth was limited to around $1.2 \mathrm{~mm}$ with a total sensitivity falloff of $16 \mathrm{~dB}$ for the entire imaging range.

In this report, we present a UHR SD-OCT system with a lownoise supercontinuum source (SC). It has an axial resolution of $2.72 \mu \mathrm{m}$ with an imaging depth of $1.78 \mathrm{~mm}$, and a lateral resolution of $5.52 \mu \mathrm{m}$ in air. We designed a customized spectrometer with low-cost off-the-shelf lenses to cover the 200-nm wavelength range with 2048 pixels. The design procedure for the spectrometer focusing optics was discussed and the performance of the customized lenses was evaluated in Zemax. Using the customized lenses, the 6-dB sensitivity falloff range was measured to be $0.89 \mathrm{~mm}$ after proper spectrometer calibration. The system had an SNR of $93.2 \mathrm{~dB}$ at a $70 \mathrm{kHz}$ line rate with 800 A-line averaging. The noise of the SC source was analyzed extensively by comparison to that of an SLD source in the same spectral range. An optimal imaging protocol was developed to mediate the impact of the excess noise from the SC in the OCT images. This system was used to examine myocardium specimens from fresh human and swine hearts. Cross-sectional OCT images and 3-D volumes were acquired ex vivo on the cardiac tissue specimens from different chambers of the hearts. Analysis of hematoxylin and eosin (H\&E) and trichrome slides showed that with increased resolution and contrast from the UHR SD-OCT system, features such as elastic fibers, Purkinje fibers, and collagen fiber bundles were observed, which were otherwise not shown in our previous work using a 1300-nm system. Correlation between the structural information provided by the OCT images, and tissue pathology was also discussed.

\section{Methods}

\subsection{Configuration of the Ultrahigh-Resolution SD-OCT System}

Figure 1 shows the schematic of the SD-OCT system. A lownoise supercontinuum laser (SuperK EXTREME, NKT Photonics) was employed to serve as a broadband light source. A set of dichroic and edge-pass filters (Thorlabs DMSP1000, FELH0650, FESH1000) was used to shape a light spectrum centered at $840 \mathrm{~nm}$ with an initial full width at half maximum (FWHM) bandwidth over $170 \mathrm{~nm}$ from the supercontinuum, which was then delivered to a free-space Michelson interferometer through a single-mode fiber (Thorlabs 780HP) and an achromatic coupler (Thorlabs AC080-010-B). The bandwidth of the source was expected to further shrink due to the cutoff wavelength of the single-mode fiber at around $730 \mathrm{~nm}$.

In the OCT imaging system, the fiber-coupled light was first collimated by an achromatic collimator (AC1, $f=19 \mathrm{~mm}$ ) and divided into sample and reference arms by a 50/50 nonpolarized beam cube (NPBC). In the sample arm, a low-NA broadband scan lens (Thorlabs LSM03-BB) was used as an imaging lens, and a pair of galvo scanners (Thorlabs GVS002) was used to transversely scan the focused beam over the sample. Dispersion mismatch was minimized by adding a block of glass (Thorlabs DCLSM03) in the reference arm. Backscattered signals from the two arms were recombined

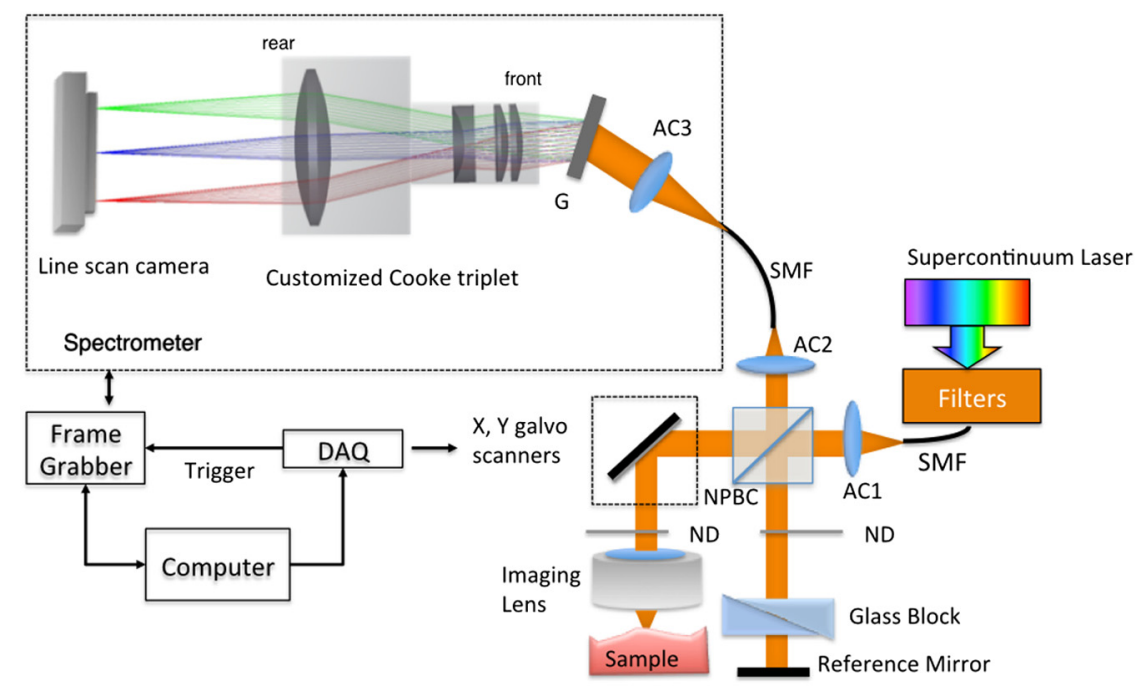

Fig. 1 Schematic of SD-OCT system. SMF, single mode fiber; AC, achromatic lenses; ND, neutral density filter; NPBC, nonpolarized beam cube; G, transmission diffraction grating. All optical components were chosen to have antireflection coating for 600 to $1000 \mathrm{~nm}$ to minimize loss. Inset: schematic of the spectrometer with customized focusing optics. 
at the NPBC and back-coupled to a single-mode fiber (Thorlabs 780HP) by an achromatic collimator (AC2, $f=$ $19 \mathrm{~mm})$. The interference signal was then measured by the home-built spectrometer.

In the spectrometer, the collimated light beam (AC3, $f=50 \mathrm{~mm}$ ) was dispersed by a transmission diffraction grating (Wasatch Photonics, $1200 \mathrm{lp} / \mathrm{mm}$ ) and focused onto a 2048pixel CCD linescan camera (e2v, AVIIVA-II EM4 BA9, 12bit) by a customized focusing lenses system. The camera, with a maximum line rate of $70 \mathrm{kHz}$, was controlled by a CameraLink framegrabber (NI, PCIe-1433) and synchronized with a pair of galvo scanners through a data-acquisition (DAQ) device (NI, PCI-6221). Image display, spectral data acquisition, and storage were all controlled by a home-built software platform written in lab view.

\subsection{Spectrometer Design and Calibration}

The spectrometer was designed to cover a wavelength range of $200 \mathrm{~nm}$ centered at $840 \mathrm{~nm}$ to accommodate the broadband source, utilizing the maximum grating efficiency at $840 \mathrm{~nm}$. In SD-OCT, the imaging depth $z_{\max }=0.25 \cdot \lambda_{0}^{2} / \delta_{\mathrm{s}} \lambda$ is dependent on the spectral sampling interval $\delta_{\mathrm{s}} \lambda{ }^{11}$ The spectral sampling interval is solely determined by the detection bandwidth. With a detector of 2048 pixels and assuming uniform sampling across the whole bandwidth $\left(\delta_{\mathrm{s}} \lambda=0.098 \mathrm{~nm}\right)$, the imaging depth was calculated to be roughly $1.8 \mathrm{~mm}$, which should be sufficient for most medical imaging applications. Since the collimated beam is angularly dispersed after passing through the transmission grating, the focusing lenses system is intended to produce a flat focal plane with minimal aberration for all wavelengths incident at different field angles. A Cooke triplet lenses system was thus designed and optimized for the desired focal length, aiming for the best focusing performance across the wavelength range of interest in ZEMAX. The schematic of the design is shown in Fig. 1. The first step of the design procedure was to determine a desired focal length, which can be estimated using a geometrical optics $\operatorname{model}^{27}$ as

$$
\begin{array}{r}
F \cdot \tan \left(\left|\sin ^{-1}\left(G \lambda-\frac{G \lambda_{\mathrm{c}}}{2}\right)-\sin ^{-1}\left(\frac{G \lambda_{\mathrm{c}}}{2}\right)\right|\right) \\
=w_{\mathrm{p}} \cdot\left|N(\lambda)-N\left(\lambda_{\mathrm{c}}\right)\right|,
\end{array}
$$

where $w_{\mathrm{p}}$ is the pixel width, $N(\lambda)$ is the wavelength-dependent pixel number assignment, $G$ is the grating constant, and $\lambda_{\mathrm{c}}$ represents the central wavelength. Using edge wavelengths (740 and $940 \mathrm{~nm}$ ), the averaged focal length was calculated from Eq. (1) to be $102 \mathrm{~mm}$, which served one of the merit functions for lens optimization. Starting with an initial design of the Cooke triplet that can be found elsewhere ${ }^{28,29}$ and restraining glass materials (N-BK and N-SF11) to match the commercial catalog spectrum, a final design of the lenses can be obtained by iterative optimization. As shown in Fig. 1, the negative lens (Thorlabs LC2679-B, N-SF11) in the center was first determined due to the limited availability in the commercial catalog. The front positive lens was split into two separate singlets (Thorlabs LA1608-B, LE1234-B, N-BK7) to best portray the optimized structure as well as to reduce the $f$-number for a higher-power transmission. The rear positive lens (Thorlabs LB1309-B, N-BK7) was chosen using the trial-and-error method based on the optimization results.

The 6-dB sensitivity falloff range $z_{6 \mathrm{~dB}} \approx 0.22 \cdot \lambda_{0}^{2} / \delta_{\mathrm{r}} \lambda$ is dependent on the spectral resolution of the spectrometer $\delta_{\mathrm{r}} \lambda{ }^{11}$ To design a 6-dB falloff range greater than $1 \mathrm{~mm}$, the minimum spectral resolution required is $0.121 \mathrm{~nm}$. The spectral resolution $\delta_{\mathrm{r}} \lambda$ can be assessed by the separation of point-spread functions (PSFs) on the imaging plane. PSF is the impulse response of an optical system. It is used to quantify the diffraction-limited optical performance in the spatial domain. The Huygens PSF is calculated based on the Huygens-Fresnel principle, and it is more accurate than the Fourier transform-based PSF calculation in the scenario of oblique incidence. As seen in Fig. 2, with a spectral resolution of $0.1 \mathrm{~nm}$, corresponding to a minimal falloff range of $1.2 \mathrm{~mm}$, the spatial separations of the PSFs at all three wavelengths were wide enough so that two PSFs could be well contained by two adjacent pixels on the detector, respectively. For 740 and $940 \mathrm{~nm}$, however, the power transmission was relatively lower and the aberration effects of the lens were more severe when compared with the central wavelength, which will impact the sensitivity falloff range as well as the $3-\mathrm{dB}$ bandwidth of the measured spectrum.

Experimentally, the spectrometer bandwidth was confirmed with the help of a set of bandpass filters. After the coupling efficiency of the camera was maximized, five bandpass filters $\left(\lambda_{\mathrm{c}}=760,800,840,880,920\right.$, and $\left.\Delta \lambda_{3 \mathrm{~dB}}=10 \mathrm{~nm}\right)$ on a filter wheel were inserted in the optical path to address the relative pixel position of the corresponding wavelength individually. The effective focal length and the respective wavelength distribution on the line sensor can be estimated based on Eq. (1). Since the pixel number was neither linear to the wavenumber $k$ nor to the wavelength $\lambda$, a two-step calibration process was carried out to generate calibration metrics for linear- $k$
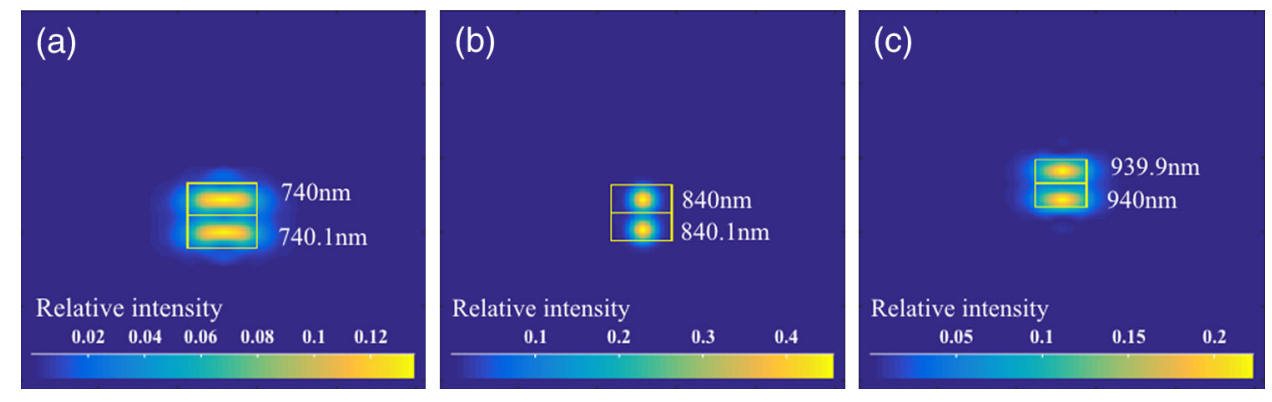

Fig. 2 PSFs for two wavelengths with a spectral separation of $0.1 \mathrm{~nm}$ at (a) 740 , (b) 840 , and (c) $940 \mathrm{~nm}$. The FWHM of the PSF was 7.4, 9.4, and $10.6 \mu \mathrm{m}$ for 740,840 , and $940 \mathrm{~nm}$, respectively. The color bar shows the relative intensity. The yellow boxes indicate the location and physical dimensions of two adjacent rectangular pixels $(14 \mu \mathrm{m} \times 28 \mu \mathrm{m})$. 
(a)

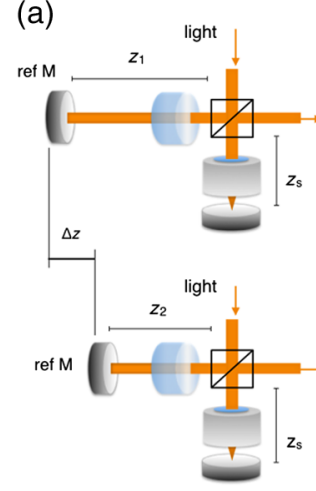

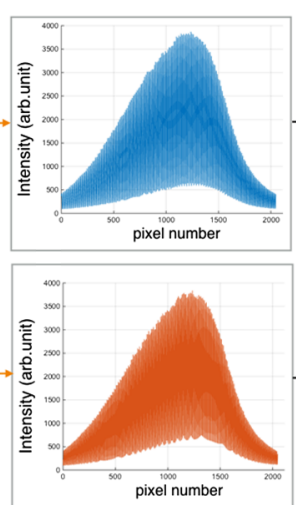

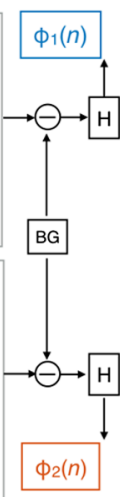

(b)

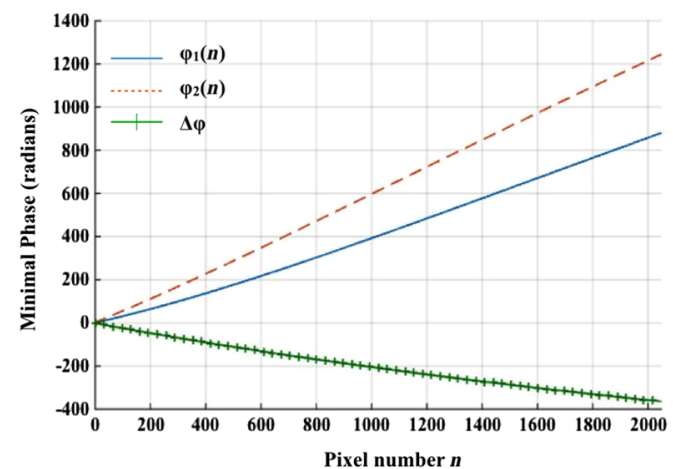

Fig. 3 (a) Spectrometer calibration process. Interferograms for two different optical path differences were generated with background subtraction. Phase information was extracted from the analytical signal constructed by Hilbert transform. (b) The minimal phases constructed from the interferogram with respect to the pixel number. The phase difference (green) of the two interference signals was fitted to a third-order polynomial as a pixel calibration metric for linear- $k$ redistribution.

distribution ${ }^{30,31}$ before inverse Fourier transform of the spectral interference signal to the spatial domain. As shown in Fig. 3(a), initially, a flat mirror was fixed at the focal plane $z_{s}$ of the sample arm to generate a set of interference fringe with a phase of

$\varphi_{1}(n)=2 k\left(z_{1}-z_{\mathrm{s}}\right)+\varphi_{\text {sys_nl }}$

when the reference mirror was at the position of $z_{1}$ [blue solid line in Fig. 3(b)]. $n$ denoted the pixel number, and the term $\phi_{\text {sys_nl }}$ represented any nonlinear dispersion introduced in the optical system. Next, the reference mirror was moved away from $z_{1}$ to a new position $z_{2}$ by a linear translation stage to generate another set of interference fringe with a phase of

$\varphi_{2}(n)=2 k\left(z_{2}-z_{\mathrm{s}}\right)+\varphi_{\text {sys_nl }}$

[red dashed line in Fig. 3(b)]. Note that the nonlinear term was not changed during this translation process; the difference between Eq. (2) and Eq. (3) reads as $\Delta \varphi(n)=2 k \Delta z$ was linear to the wavenumber $k$ [green line in Fig. 3(b)], and can be used to create a new pixel metric for linear- $k$ redistribution. Practically, the phase information of the interference signal was extracted from the analytical signal generated by Hilbert transform after background subtraction. The phase difference $\Delta \varphi(n)$ was then fitted by a third-order polynomial, which was used to create a lookup table $n^{\prime}$ for linear- $k$ interpolation of the raw spectrum.

\subsection{Postprocessing}

The calibrated source spectrum is shown in Fig. 4(a). The measured 3-dB bandwidth was around $116 \mathrm{~nm}$, which was limited by the coupling of the single-mode fiber and the low throughput of the lenses at the edge of the bandwidth. It was noted that the spectrum had a non-Gaussian profile and was cut off at $10-\mathrm{dB}$ bandwidth, which will excite spurious sidelobes in the coherence envelope after Fourier transform. Spectral correction $(\mathrm{SC})^{32}$ and apodization were therefore performed to suppress the sidelobes as well as the noise floor. In order to preserve the broad bandwidth of the spectrum, a tapered cosine window, also known as a Tukey window, was applied for apodization. The Hann window was avoided in this case since it washed out about one-third of the bandwidth, causing broadening of the axial PSF. As seen in Fig. 4(b), after applying SC and apodization, the sidelobes and noise floor around the axial PSF were suppressed significantly, while both the FHWM bandwidth of the spectrum and the PSF were preserved.

For each A-line, background subtraction and dispersion compensation were further performed to improve the axial PSF. ${ }^{33,34}$ All postprocessing steps were done separately in MATLAB ${ }^{\circledR}$ after data acquisition.
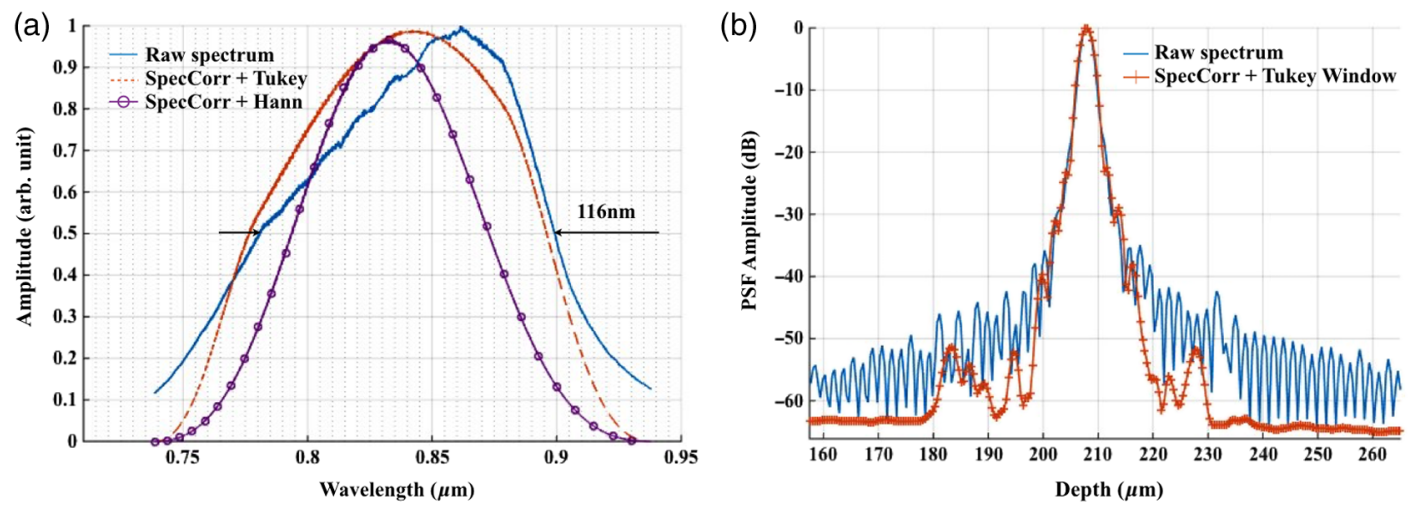

Fig. 4 (a) Spectral shape correction and apodization effects on the source spectrum. (b) The suppression of sidelobes and noise floor of the axial PSF after applying Tukey window for apodization. 


\subsection{System Characterization}

The axial resolution was defined as the FWHM of the coherence envelope, obtained by inverse Fourier transform of the interferogram taken with a single reflector at the focal plane of the sample arm. Figure 5(a) shows a typical axial PSF at $205 \mu \mathrm{m}$ path difference after background subtraction. No SC or apodization was performed. The Gaussian-fitted FWHM of the measured PSF was read as $2.72 \mu \mathrm{m}$, which was in accordance with the theoretical value of $2.68 \mu \mathrm{m}$ for the measured bandwidth of $116 \mathrm{~nm}$. After SC and apodization, the axial PSF was measured to be $2.74 \mu \mathrm{m}$. Figure 5(b) shows axial PSFs at difference depths by moving the reference mirror. It was shifted by $-113.72 \mathrm{~dB}$ to compare with the theoretical prediction (dotted curve) of the sensitivity falloff behavior, ${ }^{35}$ and the 6 - $\mathrm{dB}$ rolloff range was measured to be $0.89 \mathrm{~mm}$ (dashed line). It was less than the predicted value $(1.2 \mathrm{~mm})$, which could mainly be due to the alignment errors in the lens system. The SNR of the system was calculated as the difference between the peak amplitude in the depth profile and the averaged global noise floor between 150 and $170 \mu \mathrm{m}$ under the peak, as shown in Fig. 5(b). The sensitivity of $93.2 \mathrm{~dB}$ was read as the sum of the SNR $(63.2 \mathrm{~dB})$ and $30 \mathrm{~dB}(\mathrm{OD}=1.5)$ signal attenuation in the sample arm with $1.2 \mathrm{~mW}$ incident on the perfect reflector at the line rate of $70 \mathrm{kHz}$ with 800 A-line averaging.

The relatively high excess noise associated with SCs has been a concern for their feasibility in OCT applications. The main contribution to the noise in the OCT image stems from the power delivered by the reference arm, considering the scattered signal from the biological samples is usually negligible. For SD-OCT systems, the photoelectron noise detected by each detection unit can be written as the sum of shot noise, excess noise, and receiver noise as Eq. $(4)^{26,36}$

$\sigma^{2}=\underbrace{\left\langle n_{\mathrm{e}}\right\rangle}_{\text {shot }}+\frac{\Pi^{2}+1}{2} \underbrace{\frac{\tau_{\mathrm{coh}} N\left\langle n_{\mathrm{e}}\right\rangle^{2}}{\tau}}_{\text {excess }}+\underbrace{\sigma_{\mathrm{rec}}^{2}}_{\text {receiver }}$,

where $\left\langle n_{\mathrm{e}}\right\rangle$ is the averaged number of photoelectrons read by a detection unit, $\tau$ is the integration time of the detector, $\tau_{\text {coh }}$ is the coherence time of the light source, $N$ is the number of detection units, and $\Pi$ is the degree of polarization. The shot noise can be approximated as $\left\langle n_{\mathrm{e}}\right\rangle$ following the Poisson photon-number distribution of an ideal laser, and the excess noise expression in Eq. (4) is restricted to pure spontaneous emission source only. Since the actual pixel value PV is linearly related to the photoelectron $n_{\mathrm{e}}$ by a constant camera gain $K$ (number of electrons per analog-to-digital unit) as $n_{\mathrm{e}}=K \cdot \mathrm{PV}$, it can be used to quantitatively study the total noise of the system. Experimentally, the total noise was measured as the variance of the PV $(840 \mathrm{~nm})$ from 1000 measurements with only the reference power and the averaged number of photoelectrons as the mean PV $(840 \mathrm{~nm})$. For comparison, the same method
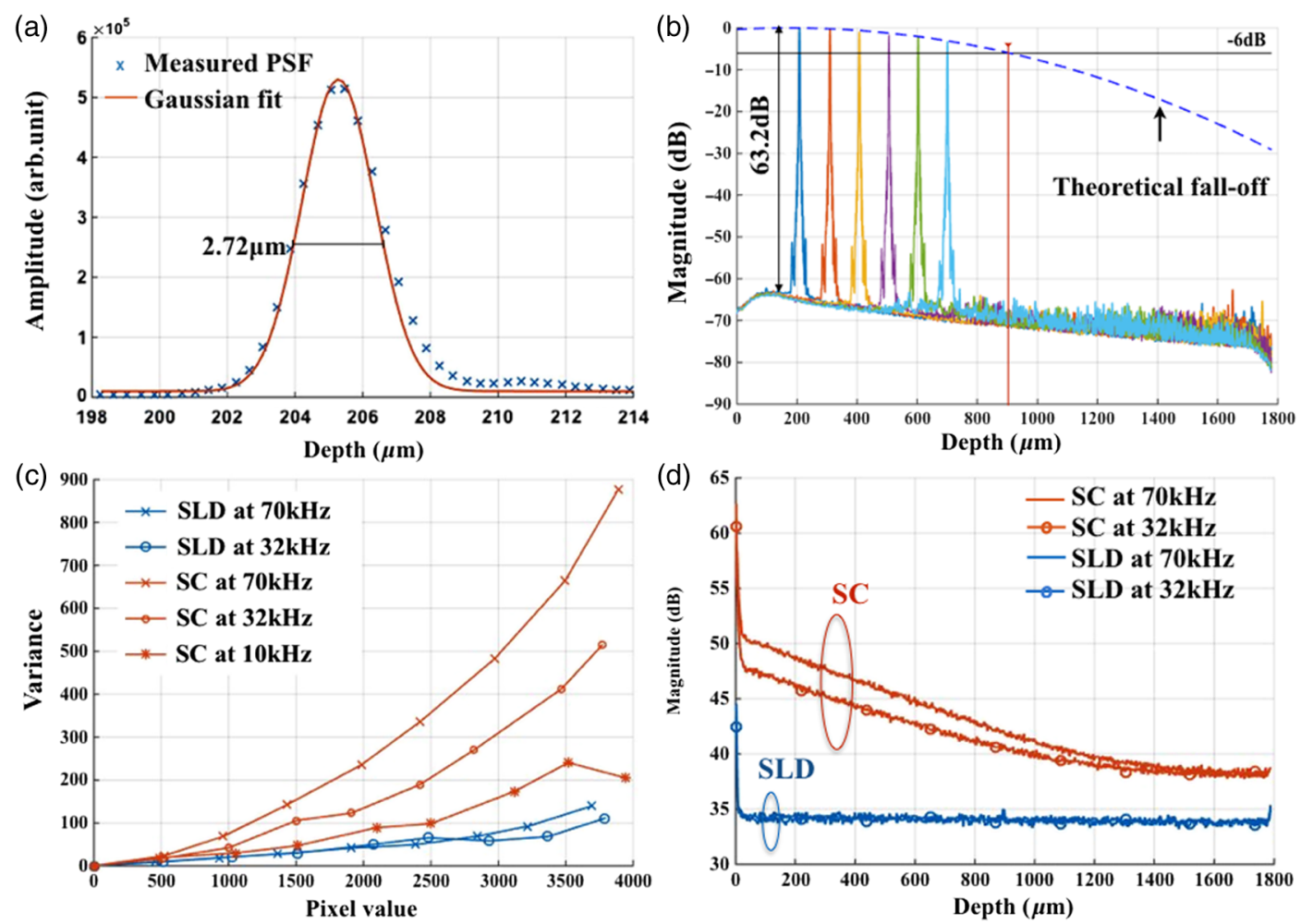

Fig. 5 (a) Measured axial PSF and Gaussian-fitted profile. (b) Sensitivity falloff measurement compared with the theoretical model. The $6-\mathrm{dB}$ falloff range was $0.89 \mathrm{~mm}$. (c) The peak intensity variances of the SC source with respect to the mean peak intensity level measured at $70 \mathrm{kHz}$ (red cross), $32 \mathrm{kHz}$ (red circle), and $10 \mathrm{kHz}$ (red star) line rate, with comparison to the SLD source at $70 \mathrm{kHz}$ (blue cross) and $32 \mathrm{kHz}$ (blue circle). (d) The noise floors in the A-line profile for SC and SLD sources at different line rates with the pixel value of the spectral peak set at $50 \%$ of the saturation level, respectively. The integration time was set as the maximum achievable value under a certain line rate. A-line averaging (1000) was applied to show a clear noise floor. 
was applied to measure the noise of an SLD source with the same center wavelength $(840 \mathrm{~nm})$ and FWHM bandwidth of $45 \mathrm{~nm}$ (INPHENIX, IPSDD0808). A bandpass filter at $840 \mathrm{~nm}$ (Thorlabs, FB840-10) was placed in the detection path to generate comparable spectra from the two different sources, and a continuously variable neutral density filter (Thorlabs, NDC50C-4-B) was employed to control the peak pixel value to a desired level. Before the optical power was turned on, the dark counts were first recorded. The variance of the dark counts, dominated by receiver noise of the detector, served as a ground noise. All the other measurements were plotted with ground noise subtraction. For all measurements, both SC and SLD were driven at their highest power levels, and the integration time was set as the maximum achievable value under a certain line rate. As seen from Fig. 5(c), the noise increased with the mean photoelectron number for both sources in general. The SC source exhibited higher intensity fluctuation compared to the SLD. At a fixed pixel level, the decrease of intensity fluctuation of the SC source at lower line rate (longer integration time) may suggest an excess-noise dominant behavior according to Eq. (4). In contrast, the noise of SLD stayed almost constant for different line rates when the averaged PV $(840 \mathrm{~nm})$ was below $70 \%$ of the saturation level, which may indicate a shotnoise dominant behavior within that power regime. Above $70 \%$ of the saturation level, the excess noise seemed to start to play a role in the SLD source as well. Furthermore, it was noted that at a line rate of $10 \mathrm{kHz}$, the intensity fluctuation of the SC source was almost comparable to that of the SLD, especially at lower intensity levels, suggesting that the noise behavior of SC may approach the shot noise limit at longer integration time. To further understand the impact of the additional noise source from the SC on the OCT image quality, Fourier domain analyses of the fluctuation in the raw spectra were performed following the method proposed by Brown et al. ${ }^{37}$ Again, 1000 spectra were acquired with reference power only for the two light sources, respectively, but this time without the bandpass filter. All Aline profiles were obtained with DC subtraction only, then averaged to show a clear noise floor. For comparison, the same processes were carried out for the SLD source. The power delivered to the spectrometer was controlled using the variable attenuation filter so that the pixel value of the spectral peak was maintained around $50 \%$ of the saturation level at different integration time settings. As shown in Fig. 5(d), it was possible to bring down the noise floor of the SC source by lowering the line rate or equivalently increasing the integration time. On the other hand, the noise floor of SLD barely changed at different line rates, which was consistent with the measurements shown in Fig. 5(c) as well. In particular, at the $32-\mathrm{kHz}$ line rate, the noise floor of the SC source in general was $5 \mathrm{~dB}$ higher than that of the $\mathrm{SLD}$, and it was around $13 \mathrm{~dB}$ higher around the DC term.

The lateral resolution of the system was determined by the optics in the sample arm. We used Zemax to simulate the sample arm optics, and the size of the diffraction-limited focal spot at the focal plane was obtained using paraxial Gaussian beam calculation. For a single-mode fiber with mode field diameter of $4.5 \sim 5.5 \mu \mathrm{m}$ at $840 \mathrm{~nm}, 1 / e^{2}$ beam diameter at the waist produced by the sample arm optics was predicted as $8.4 \sim 10.3 \mu \mathrm{m}$, corresponding to an FWHM width of $5.2 \sim 6.1 \mu \mathrm{m}$. For an entrance pupil size of $4.6 \mathrm{~mm}$, the NA of the objective was read as 0.064 , and the theoretical lateral resolution was calculated to be $5.25 \mu \mathrm{m}$ using $0.4 \cdot \lambda_{0} / \mathrm{NA}$. To experimentally verify the lateral resolution, 3-D OCT data composed of $800 \times 800 \times$ 1024 pixels covering a volume of $3 \mathrm{~mm} \times 3 \mathrm{~mm} \times 1.78 \mathrm{~mm}$ was acquired from the USAF1951 target by scanning the focused beam over the target surface. The calibration pattern can be read from the en face image and is shown in Fig. 6(a). The lateral resolution is defined as the resolving power of the last resolvable group. After analyzing the image, the last resolvable group was identified to be group 6-4, for which the averaged horizontal and vertical pixel profiles are shown in Fig. 6(b). It suggested a lateral resolution better than $5.52 \mu \mathrm{m}$, which was consistent with the theoretical prediction as well.

\subsection{Imaging Protocol}

3-D OCT datasets were generated from cardiac tissue of human hearts and swine hearts ex vivo. Human hearts $(n=7)$ were acquired from the National Disease Research Interchange (NDRI) protocol within $24 \mathrm{~h}$ of donor death, and swine hearts $(n=15)$ from Green Village Packing Company (Green Village,
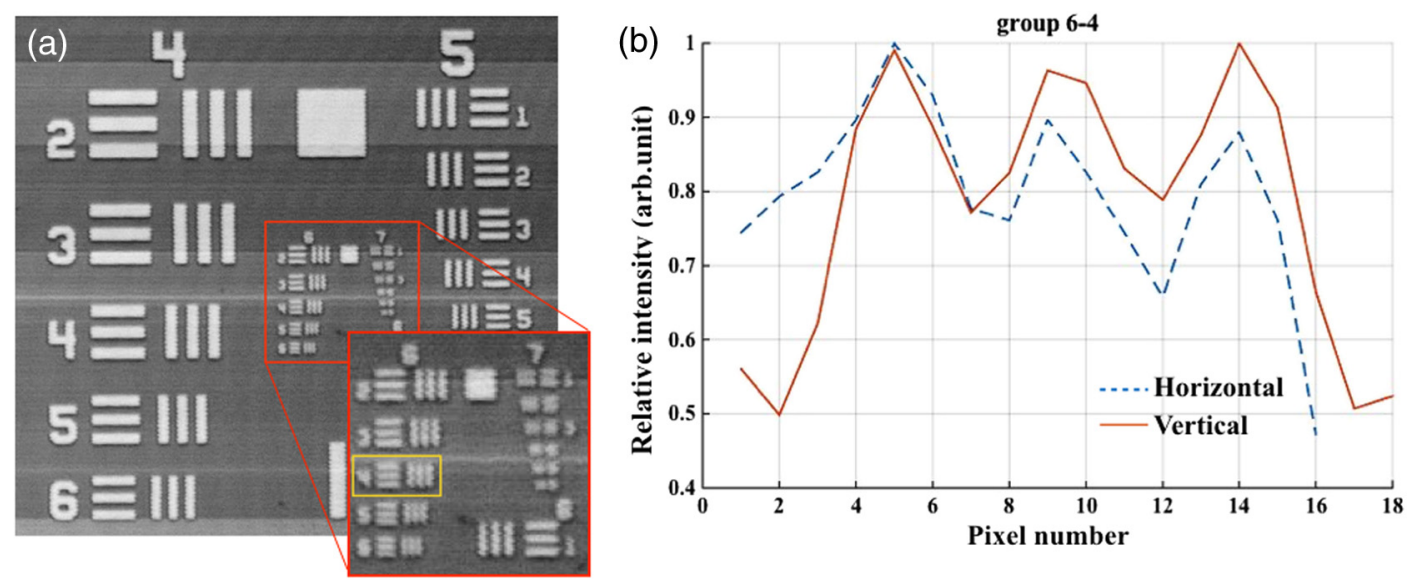

Fig. 6 Lateral resolution calibration with a USAF1951 target. (a) An averaged en face image generated from a 3-D OCT volume by extracting the orthogonal slices with respect to the depth direction. The inset shows a zoom-in view of groups 6 and 7. The last group with distinguishable line group (group 6-4) is highlighted in the yellow box. (b) The averaged pixel profiles extracted across the horizontal (blue dashed line) and vertical (red solid line) bars of group 6-4. 
New Jersey). The inclusion criteria for the first NDRI protocol are based on the following diagnosis: end stage heart failure, cardiomyopathy, coronary heart disease, and myocardial infarction. The average age of the human donors was 54, with medical history such as cardiomyopathy, hypertension, and heart failure. Upon delivery, heart samples were dissected into five parts: left atrium, right atrium, left ventricle, right ventricle, and ventricular septum. Tissue wedges were excised from each part and imaged immediately from the endocardium side, respectively. All measurements were taken within $48 \mathrm{~h}$ of the donor's death, with the incident power of $18 \mathrm{~mW}$ on the sample at a line rate of $32 \mathrm{kHz}$. The reference power was set around 30\% $50 \%$ of the pixel saturation level. These settings were identified to achieve a balanced performance regarding the image contrast, system noise, and autocorrelation artifacts. The UHR-OCT dataset was composed of $800 \times 800 \times 1024$ pixels to cover a 3 -D volume of $3 \mathrm{~mm} \times 3 \mathrm{~mm} \times 1.78 \mathrm{~mm}$.

\subsection{Histology}

The histological slices were prepared in the same orientation as a single B-scan. The thickness of each slice was set to $3 \mu \mathrm{m}$. Masson's trichrome and H\&E stains were used for histological processing. For each specimen block, six levels were generated to match the OCT images. All the histology slides were scanned by a Leica SCN400 slide scanner with $40 \times$ magnification. The digitized slides were analyzed using ImageScope (Leica).

\section{Results}

\subsection{Image Contrast}

The image contrast, known as the Michelson contrast $C$, was defined as the following:

$$
C=\frac{I_{\max }-I_{\min }}{I_{\max }+I_{\min }},
$$

with $I_{\max }$ and $I_{\min }$ representing the highest and lowest pixel intensity in the image. A single B-scan of a stack of tapes was used to measure the image contrast, as shown in Figs. 7(a) and 7(b). The image was displayed in logarithmic scale. A single A-line was extracted by averaging 20 lateral pixels, where $I_{\max }$ (83.76) and $I_{\min }$ (28.16) were taken from the pixel value of the top layer of the tape stack and that of the background right after the bottom layer, respectively, as shown in Fig. 7(b). The image contrast was calculated to be 0.49 .

The superior resolution as well as image contrast produced by the ultrahigh-resolution system at $800 \mathrm{~nm}$ was demonstrated by comparing the UHR-OCT B-scan image of a tissue specimen from a swine heart right ventricular septum in Fig. 7(c) with that taken from the Thorlabs Telesto I system $(1300 \mathrm{~nm})$ at video rate $(28 \mathrm{kHz})$ with an axial resolution of $6.5 \mu \mathrm{m}$ and lateral resolution of $15 \mu \mathrm{m}$ (in air) in Fig. 7(d). The contrast of both images was adjusted for visualization purposes. Although the penetration depth was limited in this particular UHR image due to higher scattering loss and a higher noise floor, more detailed (a)
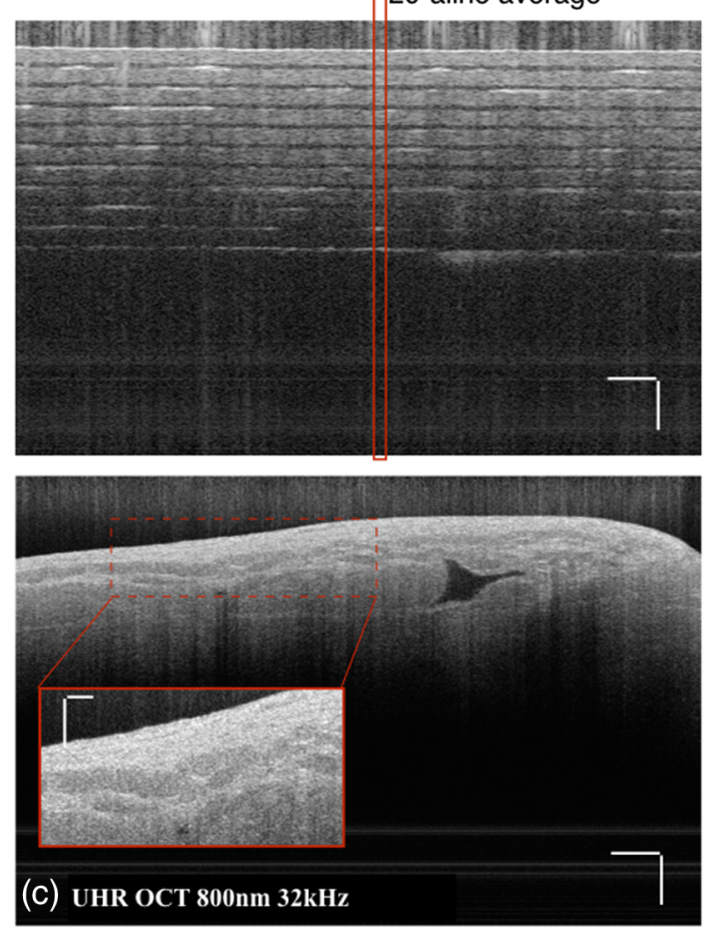

(b)
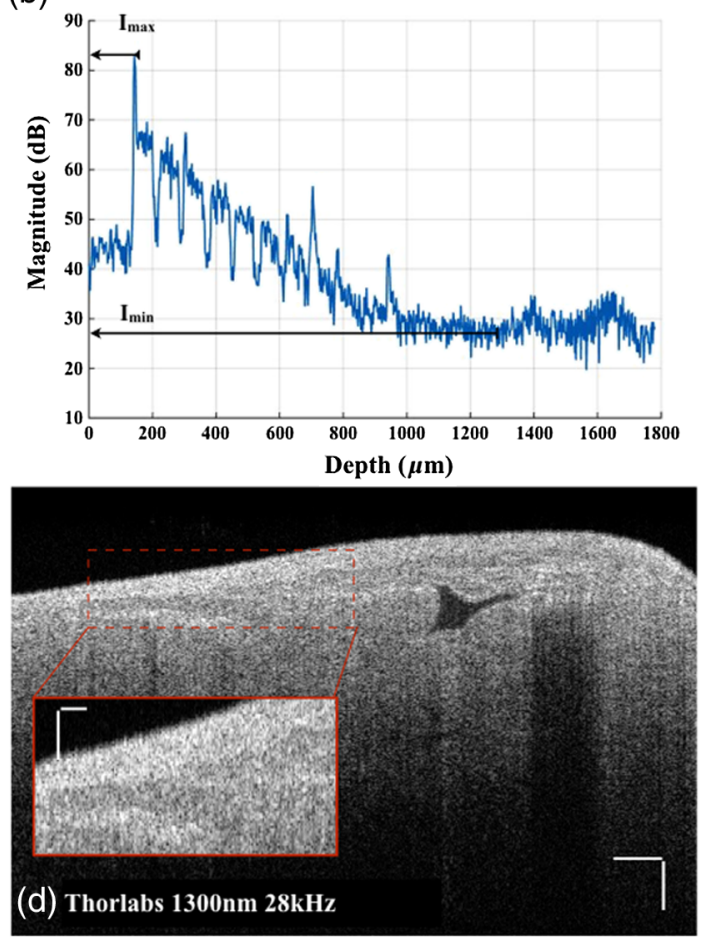

Fig. 7 (a) A single B-scan of a stack of tapes. Averaged A-line across 20 lateral pixels (red box) is shown in (b). The signal strength at the top layer of the tape stack and the background below the bottom layer was used for contrast calculation. (c) and (d) Contrast comparison between the B-scan images of the swine heart right ventricular septum acquired from (c) the UHR OCT system at $32 \mathrm{kHz}$ line rate and (d) Thorlabs Telesto system at $28 \mathrm{kHz}$ line rate, respectively. Four consecutive B-scans were averaged to show a clear image. Scale bar: $200 \mu \mathrm{m}$. Inset scale bar: $100 \mu \mathrm{m}$. 
structures were revealed in the UHR image around the endocardium layer.

\subsection{Ultrahigh-Resolution Optical Coherence Tomography Images of Heart Tissue Ex Vivo}

\subsubsection{Visualization of microstructural features in the myocardium}

Figure 8 shows different microstructural features in the myocardium of human (a)-(d) and swine hearts (e)-(f) revealed by the UHR OCT images in comparison to the corresponding H\&E slides. Collagen fiber bundles are primarily dense and long, and they run without branching, while elastic fibers are loose and branched frequently. ${ }^{38}$ In the UHR OCT images such as in Figs. 8(a), 8(c), and 8(e), the top collagen fiber layer was delineated by a higher intensity of back-scattered signal, and the elastic fibers [Fig. 8(c)] were more branched with less contrast. The honeycomb-like tissue found in Fig. 8(a) was adipose tissue. Normal hearts rarely have adipose in the myocardium, while a higher occurrence of mature adipocytes is reported to have associations with healed myocardial infarction. ${ }^{39}$ It should be noted that the endocardium layer of the human heart is generally thinner than that of the swine heart. The endocardium layers in Figs. 8(a) and 8(c) showed a thickened appearance, which could correspond to some specific types of pathology such as endocardium fibroelastosis or subendocardium fibrosis from ventricular dilation. Moreover, the H\&E slide in Fig. 8(d) showed possible diffused fibrosis in the human left ventricular septum. The Purkinje fiber is present in the subendocardium, as indicated in the UHR OCT image in Fig. 8(e). It is well correlated with the corresponding H\&E histology slide, as in Fig. 8(f), where Purkinje fibers are stained with a paler pink color. The contours of the Purkinje fiber bundles were clearly captured by the UHR OCT image. The en face images were generated parallel to the top interface with a pixel offset $d$ along depth, where different tissue types were also clearly revealed.

One typical 3-D UHR OCT volume generated from the human right ventricular septum is presented in Fig. 9(a) and Video 1. Based on our previously reported surface detection algorithm (13), a series of en face images were extracted parallel to the tissue surface along the axial direction. As seen in Figs. 9(b)-9(d), features such as collagen fiber bundles [Fig. 9(b)], adipose tissue [Fig. 9(c)], and myofibers [Figs. 9(c)9(d)] were visualized from individual en face images at different depths. In particular, the nonuniform change of myofiber tract orientation can be noticed from different en face images along the depths. This may be the cause of the irregular birefringence artifacts that appeared in the B-scan image.

\subsubsection{Birefringence artifacts in the heart tissue}

The polarization state as well as the optical path of incident light is altered after passing through the biological tissues
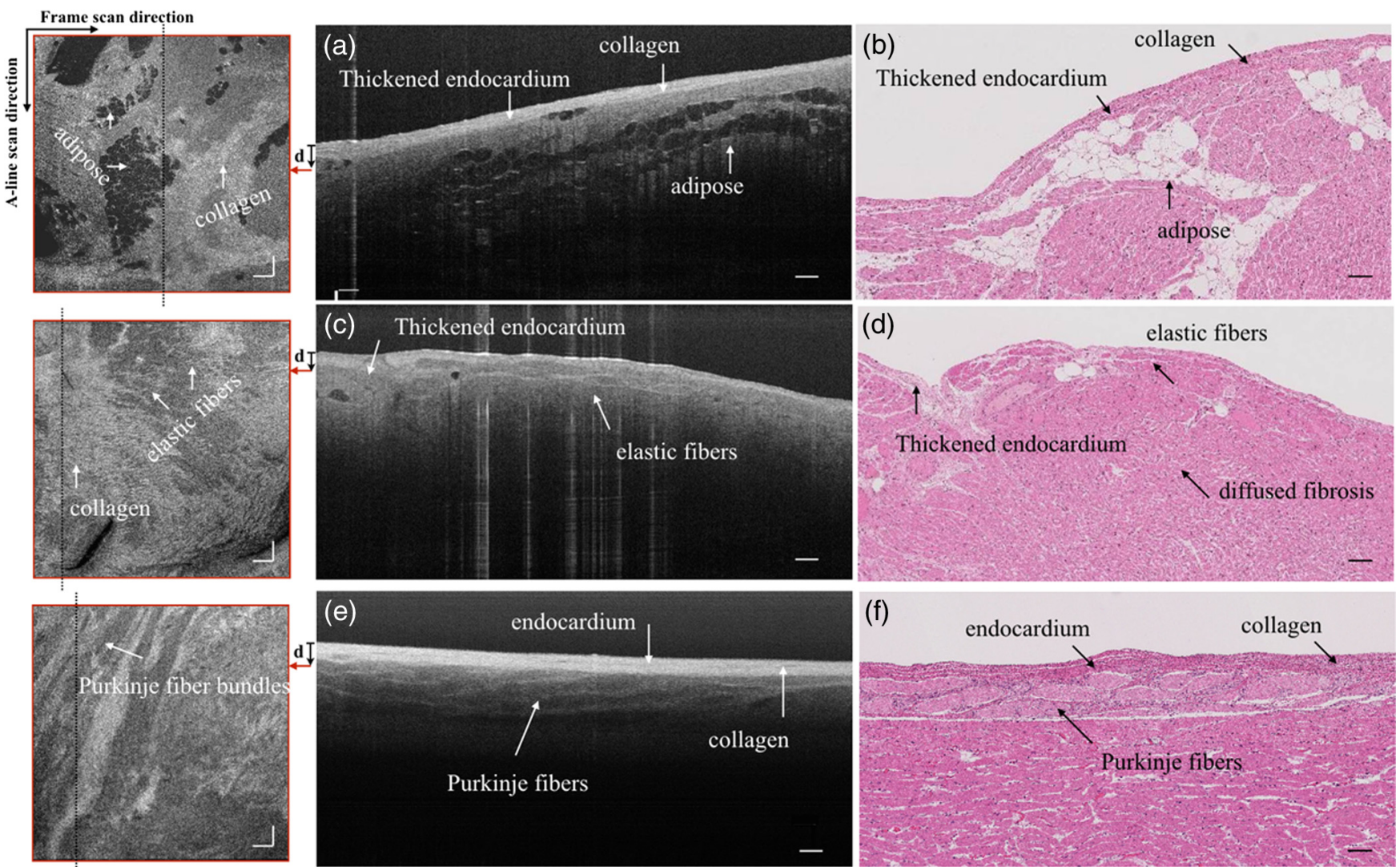

Fig. 8 UHR OCT images (a), (c), and (e) in comparison with corresponding histology slides (b), (d), and (f). The images were acquired from (a) to (d) left ventricular septum of human hearts and (e) and (f) right ventricular septum of swine hearts. Insets: en face images taken parallel to the top surface with a pixeloffset $d$ in depth. (a) $d=51$; (b) $d=31$; (c) $d=30$. B-scan average: 4 . Scale bar: $100 \mu \mathrm{m}$. Inset scale bar: $200 \mu \mathrm{m}$. 

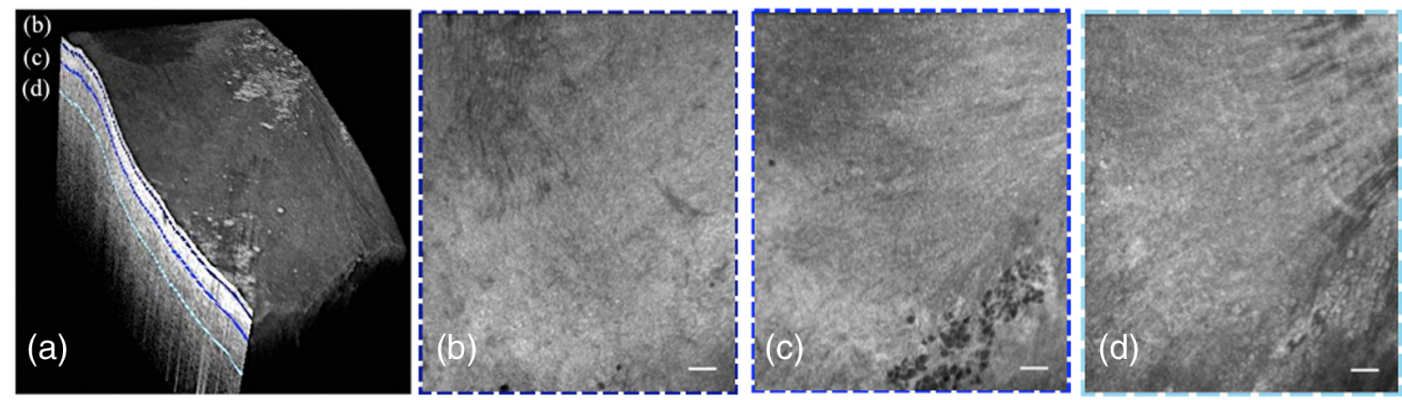

Fig. 9 (a) 3-D visualization of UHR OCT volume taken from the right ventricular septum of human heart (Video 1). (b)-(d) En face images parallel to the top surface of the 3D volume from top to bottom. The intensity was normalized for each en face image for visualization. (b) Collagen fiber bundles; (c) mixture of adipose tissue and myofibers; (d) myofibers. Scale bar: $250 \mu \mathrm{m}$. (Video 1, MP4, 1.88 MB) [URL: http:// dx.doi.org/10.1117/1.JBO.21.6.061006.1].

that manifest optical birefringence, and causes the birefringence artifact to occur in the OCT images. The appearance of the birefringence artifact, on the other hand, may provide qualitative information on tissue properties. For example, two UHR OCT images in Figs. 10(a) and 10(d) were taken from the right ventricular septum of a human heart sample with the presence of the chordae tendineae as depicted in the corresponding trichrome slides shown in Fig. 10(b) and 10(e). The chordae tendineae are mainly composed of bundled fibrous tissue that also manifests optical birefringence. The birefringence artifact in the chordae tendineae showed a "double band" appearance, while in the myocardium it appeared as a "single band." Moreover, the trichrome stain also captured the fibrosis regions in both of the slides. When light interacts with the disorganized collagen in the fibrosis region, it quickly becomes depolarized, thus the birefringence artifact disappeared, causing disruption in the otherwise continuous "band." The heterogeneity in the myocardium was successfully depicted in the UHR OCT images, while it was not quite as pronounced in corresponding H\&E slides as in Figs. 10(c) and 10(f).

\subsubsection{Alignment of polarization to reduce birefringence artifact in the healthy heart tissue}

Normal myocardium contains well-aligned bundles of cardiomyocytes with homogeneous distribution. ${ }^{40}$ Without controlling the polarization of light, the detection is susceptible to birefringence artifacts, which will affect the penetration depth of the signal. To minimize the impact of the birefringence artifact on the penetration depth in the normal myocardium, we introduced a half-wave plate into the sample arm to control the alignment of the polarization state of incident light with respect to the underlying myocardial fiber orientation. No noticeable change was found in either the bandwidth or the axial resolution after inserting the half-wave plate to light path. The results are shown in Fig. 11. Initially, the fast axis of the half-wave plate was set to $0 \mathrm{deg}$, and the birefringence artifact was recognized as a black "band" in the OCT B-scan of the tissue specimen from the right ventricular septum of the swine heart [Fig. 11(a)]. By rotating the fast axis of the half-wave plate to $20 \mathrm{deg}$, it was possible to shift the "band" downward [Fig. 11(b)]. Specifically, we extracted the A-line profile averaged across 20 transverse
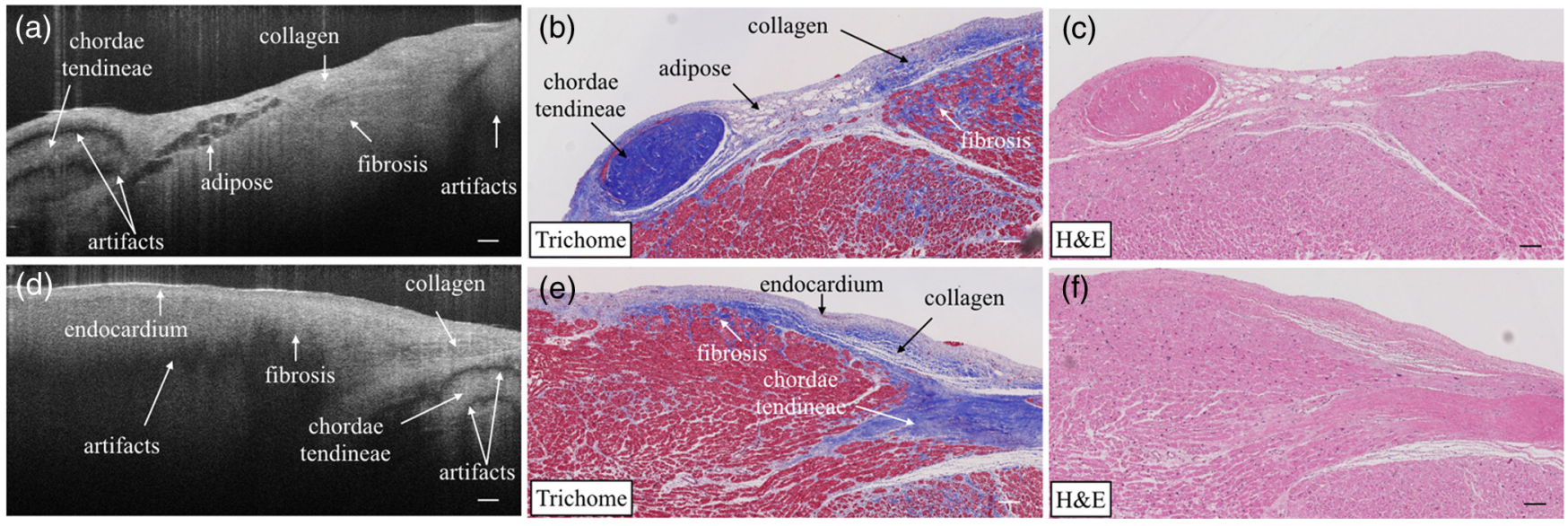

Fig. 10 The appearance of the birefringence artifact may provide qualitative information regarding tissue content. (a) and (d) UHR OCT images of the right ventricular septum of a human heart sample. The birefringence artifact showed a different appearance in the chordae tendineae as compared to that in the myocardium. The disruption of the band indicated heterogeneity (fibrosis) in the myocardium. (b) and (e) The corresponding trichrome stain slides. (c) and (f) The corresponding H\&E stain slides. Scale bar: $100 \mu \mathrm{m}$. 

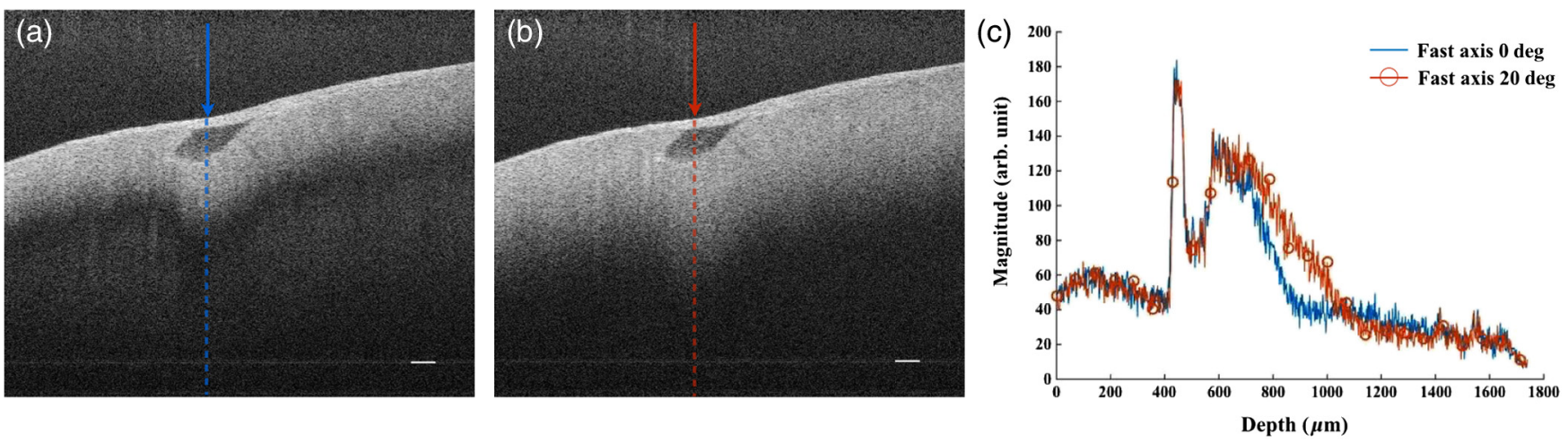

Fig. 11 Penetration depth and signal attenuation with respect to incident polarization state alignment. (a) Fast-axis of half-wave plate was set to 0 deg. (b) Fast-axis of half-wave plate was set to 20 deg. (c) 20 pixel averaged A-line profiles taken at the regions marked with arrows in (a) and (b). Scale bar: $100 \mu \mathrm{m}$.

pixels at the same location (marked) in both OCT B-scan images in Fig. 11(c). It can be seen that the signal attenuation due to birefringence was mediated and the signal penetration was thus extended. The maximum penetration depth was measured to be $0.42 \mathrm{~mm}$.

\section{Discussion}

To our best knowledge, this paper reported the first high-resolution myocardial OCT imaging results of fresh human hearts. Myocardial imaging requires high resolution and large imaging depth to visualize both the endocardium and the myocardium, even in the presence of a thickened endocardium observed during remodeling. We showed that by careful design and calibration of the spectrometer of the SD-OCT system, it is possible to achieve an ultrahigh axial resolution $(2.72 \mu \mathrm{m}$ in air $)$ and a large imaging depth $(1.78 \mathrm{~mm})$ with a sufficient $6-\mathrm{dB}$ falloff range $(0.89 \mathrm{~mm})$ at the same time. In particular, we proposed a way to design a cost-efficient focusing lens of the spectrometer, obtaining a tuning wavelength range with optimal optical performance. The lateral resolution, on the other hand, was compromised to allow for an appreciable imaging depth of the myocardium. In addition, there have been limited demonstrations of commercial SC sources in UHR-OCT for medical imaging application at the 800-nm optical window. ${ }^{19,20,41}$ The major concern lies in the excessive noise associated with SC generation. We showed that by controlling the line rate as well as the reference power on the detector, it was possible to maintain appreciable image quality with the abundant optical power input to the sample arm. The UHR OCT system was able to resolve different tissue types in the myocardium. It especially enabled the visualization of microstructures such as Purkinje fibers in the subendocardium, which were never seen by the conventional $1300 \mathrm{~nm}$ system. Moreover, the system has great potential to be converted to in vivo configuration, opening the door to addressing the clinical difficulties in the current EMB procedure for surveillance of organ rejection after heart transplant.

\subsection{Ultrahigh-Resolution SD-OCT Design Consideration and Postprocessing Methods}

SD-OCT is known to have a sensitivity advantage over its timedomain counterpart by dispersing the signal onto N-pixel detection channels to effectively reduce the noise equivalent bandwidth, ${ }^{24}$ but the finite spectral sampling interval and spectral resolution ultimately limit its feasibility at larger imaging depths. ${ }^{35}$ This makes broadband spectrometer design especially challenging, since one has to balance between the spectrometer bandwidth and the imaging depth to achieve high axial resolution without limiting the sensitivity at deeper penetration depths in the tissue. In this report, we showed that it is possible to make a low-cost Cooke triplet lenses system using off-the-shelf spherical lenses with optimized optical performance to meet the requirements of both bandwidth and spectral resolution. There are several advantages to choosing the modified Cooke triplet configuration. The diversity of the spherical singlets makes it possible to tune the effective focal length of the lenses system as well as the spectrometer bandwidth when compared with a single achromatic lens or an achromatic doublet. On the other hand, the triplet design can produce a flat focal plane with optimal focusing performance for a wide field of view. This is desired for spectrometers since the grating will angularly disperse the wavelengths to different field angles before entering the lens. By carefully choosing the lenses of the triplet, it is possible to provide the required bandwidth coverage with considerably good spectral resolution. One drawback of the presented design was its bulky package, since the total axial length was longer than $10 \mathrm{~cm}$. This was mainly caused by the choice of the rear positive lens. Theoretically, the lenses system could be made more compact if the thickness and diameter of the rear lens were more constrained.

The spectrometer reported in this study has 2048 pixels to cover $200 \mathrm{~nm}$, achieving an imaging depth of $1.78 \mathrm{~mm}$ and 6-dB falloff range of $0.89 \mathrm{~mm}$, which are sufficient for imaging myocardial tissues with strong scattering at the 800 -nm spectral window in a confocal setting. The sensor in our system has a rectangular pixel size $(14 \mu \mathrm{m} \times 28 \mu \mathrm{m})$ to provide a larger detection area, which effectively increases the coupling efficiency and benefits the system with a higher SNR. It should be noted that the actual bandwidth $(\sim 116 \mathrm{~nm})$ resolved on the spectrometer was narrower compared with that of the input source spectrum $(>170 \mathrm{~nm})$, which can be attributed to the lower throughput of the focusing lenses as well as the lower coupling efficiency of the single mode fiber at the band edges. The axial resolution can be further improved by expanding the FWHM bandwidth on the line detector with a better focusing lenses design.

Proper spectrometer calibration is required to maintain a relatively constant PSF over the depth and desired sensitivity falloff range. We showed that the pixel assignment of the 
spectrometer was calibrated into linear- $k$ distribution by simply translating the reference mirror without the need of any additional equipment. The extracted phase difference was further fitted by a third-order polynomial to eliminate the phase noise, which otherwise will cause an increase in the noise floor due to interpolation error. Other calibration methods such as utilization of standard narrow spectral line emission from calibration lamps ${ }^{21}$ and iteration-based numerical calibration ${ }^{41}$ can also be applied to achieve the same results.

It should be noted that the customized spectrometer only covers $10-\mathrm{dB}$ bandwidth of the source spectrum. It was necessary to apply apodization to suppress the sidelobes excited by discrete Fourier transform. While the Hann window excels in sidelobe suppression, it also results in a broadened axial PSF in the depth profile and thus degrades the system capability of ultrahigh-resolution imaging. A tapered cosine window (Tukey window) with optimized settings was efficient in terms of bandwidth preservation as well as sidelobe suppression and was thus adopted in this study as an alternative option. Ideally, the spectrometer range could be designed to accommodate the full source spectrum, so that apodization using the Hann window may be more efficient. Nonetheless, it will compromise the imaging depth and probably shrink the sensitivity falloff range by increasing the spectral sampling interval. Another way to accommodate the full spectrum is to use a longer line sensor with more pixels, which necessarily accompanies higher cost.

\subsection{Noise of the Supercontinuum Source}

The key of the UHR OCT system is the broadband light source. Compared with other broadband sources such as multiplexed SLD and thermal light, SC lasers are beneficial for ultrahigh-resolution OCT applications owing to the higher output power and wider wavelength range confined in one single transverse mode. It also provides opportunities for functional extensions of the OCT system. However, the high intensity fluctuation associated with the SC generation remains a concern for their feasibility in OCT applications. We experimentally evaluated the noise of the SC-based system and compared it with that of the SLD-based system. It was shown that the SC source exhibited a higher noise level than SLD. However, increasing the integration time and controlling the reference power can efficiently suppress the noise of the SC source. In Particular, the noise on the single detector unit can be brought marginally beyond the shot noise limit when the line rate is down to $10 \mathrm{kHz}$ with the reference power well controlled below $50 \%$ of pixel saturation. It indicates that if the reference power detected by the spectrometer were well controlled, the SC-based OCT system could perform in the shot-noise limit in a situation where a high imaging speed is not required. Very recently, Yuan et al. also reported a similar operation strategy for controlling the SC noise in an OCT application. ${ }^{42}$

To analyze the noise in the spatial domain, the entire source spectra were Fourier transformed to obtain the noise distribution in the depth profile. At a line rate of $32 \mathrm{kHz}$, the noise floor of the SC source was about $5 \mathrm{~dB}$ higher than that of the SLD when the spectral peak value was fixed to around $50 \%$ of the pixel saturation. This was expected from Parseval's theorem, given the fact that the signal provided by the SC source has a broader bandwidth than that of the SLD. For the SC source, there was approximately a 13-dB lift of noise floor observed from the DC side. Considering that the SC source can provide at least fourfold power to the sample arm, it means the interference signal strength provided by the SC will also be higher compared to the SLD. When imaging weakly scattering media such as the myocardium, the increase in the sample power will help enhance the fringe visibility as well as the spectral density in the depth profile, without affecting the reference power domination of the total noise. Thus, the additional noise of the SC source was not expected to jeopardize the OCT image contrast. Even though the noise performance of the SC source still requires improvement to reach the shot-noise limited regime, the current state-ofthe-art SC source is feasible for myocardial OCT imaging. Furthermore, Brown et al. ${ }^{37}$ also pointed out that it was possible to improve the OCT image contrast by moving the image area away from the higher part (close to DC) to the lower part of the noise floor, which was referred to as the extended depth imaging technique. A-line or B-scan averaging can further enhance the image contrast as well.

\subsection{Ultrahigh-Resolution SD-OCT for Human Myocardial Imaging}

For normal human heart tissue, the endocardium layer is between 7 to $20 \mu \mathrm{m}$ in thickness, mainly comprised of dense collagen fibers, loose connective tissue, and squamous epithelial tissue. Therefore, the axial resolution of the UHR-OCT system ( $1.9 \mu \mathrm{m}$ in tissue with $n=1.4$ ) is sufficient for endocardium, myocardium, and visualizing changes due to remodeling. Due to the superior axial resolution and stronger backscattering of light with shorter wavelengths, different tissue contents such as collagen fiber bundles and adipose tissue in the myocardium were well depicted in the images produced from the UHR SDOCT system. Moreover, it successfully delineated microstructural features such as Purkinje fibers in the endomyocardial tissue, which were otherwise buried in the images produced by the conventional 1300-nm OCT system. Delineation of these features can add valuable information for tissue classification algorithms to facilitate automated image analysis ${ }^{43}$ in the high-speed data acquisition scenario. UHR-OCT imaging of the myocardium also allows visualization of the macroscopic structural information of the tissue content, which may correlate with the function of the myocardium. Collagen fiber bundles are responsible for the mechanical integrity of many organ systems $^{44,45}$ and can be resolved in the UHR-OCT images of the human and swine myocardium. However, subcellular assessment of microstructures, such as imaging individual collagen fibers, will require imaging modalities with higher optical resolution such as dark-field and/or SHG microscopy. ${ }^{46-48}$ Although images from the UHR OCT system may not reach the resolution of standard confocal microscopy and the contrast may be compromised due to the higher noise floor associated with SC generation, it is capable of providing a wealth of information for in vivo real-time imaging, addressing a need that is not currently covered by standard medical imaging modalities.

The structural anisotropy of the myocardial tissue is responsible for the birefringence artifact appearing in the myocardium. In healthy swine hearts, the cardiomyocytes are well aligned and the birefringence band was expected to be uniform, while in human hearts from donors of previously diagnosed cardiovascular diseases, it appeared to be disrupted. Figures 12(a) and 12(b) show some examples of irregular birefringence bands in the human right ventricular septum. The irregular appearance of the bands may be associated with differentiation in the myocardial fiber sheets, nonuniformity of local 

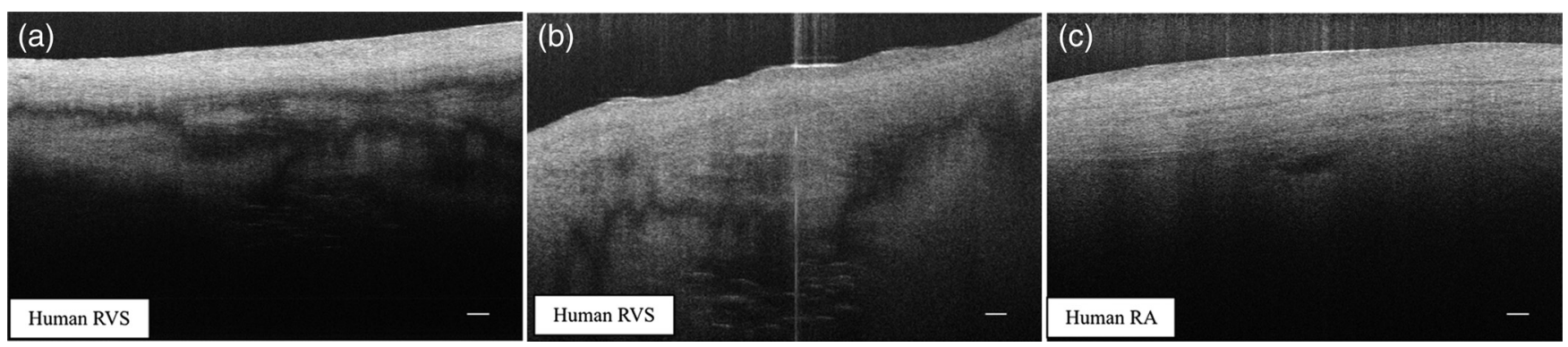

Fig. 12 (a) and (b) Irregular polarization dependent bands shown in the UHR OCT images of tissue specimen taken from the right ventricular septum of human hearts. This may be associated with the heterogeneity in the tissue composition. (c) UHR OCT image of the right atrium of human heart. The thick collagen layer depolarizes the light as it passes through the tissue and no birefringence artifact was shown. Scale bar: $100 \mu \mathrm{m}$.

myocardial fiber orientation, and/or heterogeneity in the myocardium, the details of which should be further investigated using functional OCT imaging such as PS-OCT. Moreover, the endocardium thickness was also expected to have correlations with the polarization state of the light. For the normal heart where the endocardium layer is very thin, the collagen impact on the polarization state of light can be ignored. However, in the progression of some heart diseases, the collagen content within the myocardium can increase, and a thickened endocardium appearance may suggest a sign of fibrosis. As a result, the increase of collagen fibers may eventually depolarize the light and cause a disappearance of the birefringent artifact. For example, Fig. 12(c) shows the thickened endocardium layer in the right atrium of a human heart with high collagen content. The disappearance of the birefringence artifacts in the myocardium may be attributed to the depolarization of light after multiple elastic scattering in the disorganized collagen fibers or the reduced signal penetration suffered from high scattering loss. Last but not least, our previous work on near-infrared spectroscopy has shown that this window has spectra differences for important chromophores in cardiac tissue such as hemoglobin, myoglobin, lipid, and water. ${ }^{4}$ Due to the rich spectroscopic information contained in the 800-nm regime, functional extension of the standard OCT imaging, such as spectroscopic OCT, will be beneficial as well.

\section{Conclusion}

We present a UHR SD-OCT system with a low-noise SC source that has an axial resolution of $2.72 \mu \mathrm{m}$ and a lateral resolution of $5.52 \mu \mathrm{m}$ in air. We designed a customized spectrometer with a 200-nm wavelength range to accommodate the spectral shaped supercontinuum output with an FWHM bandwidth of $116 \mathrm{~nm}$ centered at $840 \mathrm{~nm}$. The $6-\mathrm{dB}$ sensitivity falloff range was $0.89 \mathrm{~mm}$ after calibration. The optimal design and proper calibration of the spectrometer enable high axial resolution with a long imaging depth and a sufficient 6-dB falloff range for myocardial imaging. The noise of the SC source was studied extensively, and an optimal imaging protocol $(32 \mathrm{kHz}$ line rate, with peak reference power set between $30 \%$ and $50 \%$ of pixel saturation level) was proposed to suppress the noise level in the OCT image when using the SC source. Cross-sectional images and 3 -D volumes were acquired ex vivo from the endocardium side on tissue specimens of fresh human and swine hearts. Analysis of $H \& E$ and trichrome slides showed that with the increased resolution provided by the UHR OCT system, fine features such as elastic fibers, adipose, Purkinje fibers, and collagen fiber bundles were successfully delineated. Moreover, polarization-related features may provide additional information on tissue composition and can potentially be used for pathological assessment as well. UHR-OCT images provided rich structural information of the myocardium, and can potentially be employed to study the structure and function relation in the myocardium.

\section{Acknowledgments}

The authors would like to thank Mr. Rajinder Singh-Moon for sample preparation, Mr. Thomas Feuchter from NKT Photonics, and Mr. Yuye Ling for helpful discussion. This work was funded by the following sources: NIH 1DP2HL127776-01 (CPH), Columbia University Research Initiatives for Science and Engineering (RISE) (CPH), and the Feldstein Medical Foundation $(\mathrm{CPH})$.

\section{References}

1. J. N. Cohn, R. Ferrari, and N. Sharpe, "Cardiac remodeling-concepts and clinical implications: a consensus paper from an international forum on cardiac remodeling," J. Am. Coll. Cardiol. 35(3), 569-582 (2000).

2. B. J. D. Boukens et al., "Developmental basis for electrophysiological heterogeneity in the ventricular and outflow tract myocardium as a substrate for life-threatening ventricular arrhythmias," Circ. Res. 104(1), 19-31 (2009).

3. N. Koitabashi and D. A. Kass, "Reverse remodeling in heart failuremechanisms and therapeutic opportunities," Nat. Rev. Cardiol. 9(3), 147-157 (2012).

4. R. P. Singh-Moon, C. C. Marboe, and C. P. Hendon, "Near-infrared spectroscopy integrated catheter for characterization of myocardial tissues: preliminary demonstrations to radiofrequency ablation therapy for atrial fibrillation," Biomed. Opt. Express 6(7), 2494-2511 (2015).

5. L. T. Cooper et al., "The role of endomyocardial biopsy in the management of cardiovascular disease: a scientific statement from the American Heart Association, the American College of Cardiology, and the European Society of Cardiology," Circulation 116(19), 2216-2233 (2007).

6. A. M. From, J. J. Maleszewski, and C. S. Rihal, "Current status of endomyocardial biopsy," Mayo Clin. Proc. 86(11), 1095-1102 (2011).

7. E. X. Wu et al., "MR diffusion tensor imaging study of postinfarct myocardium structural remodeling in a porcine model," Magn. Reson. Med. 58(4), 687-695 (2007).

8. E. E. Konofagou, J. D'hooge, and J. Ophir, "Myocardial elastographya feasibility study in vivo," Ultrasound Med. Biol. 28(4), 475-482 (2002).

9. M. Nahrendorf et al., "High-resolution imaging of murine myocardial infarction with delayed-enhancement cine micro-CT," AJP: Heart Circ. Physiol. 292(6), H3172-H3178 (2007). 
10. D. Huang et al., "Optical coherence tomography," Science 254(5035), 1178-1181 (1991).

11. W. Drexler and J. G. Fujimoto, Optical Coherence Tomography: Technology and Applications, Springer Science \& Business Media, New York (2008).

12. C. P. Fleming et al., "Quantification of cardiac fiber orientation using optical coherence tomography," J. Biomed. Opt. 13(3), 030505 (2008).

13. Y. Gan and C. P. Fleming, "Extracting three-dimensional orientation and tractography of myofibers using optical coherence tomography," Biomed. Opt. Express 4(10), 2150-2165 (2013).

14. C. P. Fleming et al., "First in vivo real-time imaging of endocardial RF ablation by optical coherence tomography," J. Innovations Cardiac Rhythm Manage. 2, 199-201 (2011).

15. B. Povazay et al., "Submicrometer axial resolution optical coherence tomography," Opt. Lett. 27(20), 1800-1802 (2002).

16. A. R. Tumlinson et al., "In vivo ultrahigh-resolution optical coherence tomography of mouse colon with an achromatized endoscope," J. Biomed. Opt. 11(6), 064003 (2006).

17. B. Cense et al., "Ultrahigh-resolution high-speed retinal imaging using spectral-domain optical coherence tomography," Opt. Express 12(11), 2435-2447 (2004).

18. H. Wang, C. P. Fleming, and A. M. Rollins, "Ultrahigh-resolution optical coherence tomography at $1.15 \mu \mathrm{m}$ using photonic crystal fiber with no zero-dispersion wavelengths," Opt. Express 15(6), 3085-3092 (2007).

19. L. Liu et al., "Imaging the subcellular structure of human coronary atherosclerosis using micro-optical coherence tomography," Nat. Med. 17(8), 1010-1014 (2011).

20. R. Yadav et al., "Micrometer axial resolution OCT for corneal imaging," Biomed. Opt. Express 2(11), 3037-3046 (2011).

21. C. S. Cheung, M. Spring, and H. Liang, "Ultra-high resolution Fourier domain optical coherence tomography for old master paintings," Opt. Express 23(8), 10145-10157 (2015).

22. A. R. Tumlinson et al., "Endoscope-tip interferometer for ultrahigh resolution frequency domain optical coherence tomography in mouse colon," Opt. Express 14(5), 1878-1887 (2006).

23. J. Xi et al., "Diffractive catheter for ultrahigh-resolution spectral-domain volumetric OCT imaging," Opt. Lett. 39(7), 2016-2019 (2014).

24. J. F. de Boer et al., "Improved signal-to-noise ratio in spectral-domain compared with time-domain optical coherence tomography," Opt. Lett. 28(21), 2067-2069 (2003).

25. W. Drexler et al., "In vivo ultrahigh-resolution optical coherence tomography," Opt. Lett. 24(17), 1221-1223 (1999).

26. R. Leitgeb, C. Hitzenberger, and A. Fercher, "Performance of Fourier domain vs. time domain optical coherence tomography," Opt. Express 11(8), 889-894 (2003).

27. B. Park et al., "Real-time fiber-based multi-functional spectral-domain optical coherence tomography at $1.3 \mu \mathrm{m}$," Opt. Express 13(11), 39313944 (2005).

28. M. Laikin, Lens Design, CRC Press, Boca Raton, Florida (2006).

29. W. J. Smith, Modern Lens Design, McGraw-Hill, New York (2005).

30. S. Makita, T. Fabritius, and Y. Yasuno, "Full-range, high-speed, highresolution $1-\mu \mathrm{m}$ spectral-domain optical coherence tomography using BM-scan for volumetric imaging of the human posterior eye," Opt. Express 16(12), 8406-8420 (2008).

31. X. Yu et al., "High-resolution extended source optical coherence tomography," Opt. Express 23(20), 26399-26413 (2015).

32. R. Tripathi et al., "Spectral shaping for non-Gaussian source spectra in optical coherence tomography," Opt. Lett. 27(6), 406-408 (2002).

33. M. Wojtkowski et al., "Ultrahigh-resolution, high-speed, Fourier domain optical coherence tomography and methods for dispersion compensation," Opt. Express 12(11), 2404-2422 (2004).

34. N. Nassif et al., "In vivo human retinal imaging by ultrahigh-speed spectral domain optical coherence tomography," Opt. Lett. 29(5), 480-482 (2004).
35. N. Nassif et al., "In vivo high-resolution video-rate spectral-domain optical coherence tomography of the human retina and optic nerve," Opt. Express 12(3), 367-376 (2004).

36. S. Sunghwan et al., "Characterization and analysis of relative intensity noise in broadband optical sources for optical coherence tomography," IEEE Photonics Technol. Lett. 22(14), 1057-1059 (2010).

37. W. J. Brown, S. Kim, and A. Wax, "Noise characterization of supercontinuum sources for low-coherence interferometry applications," J. Opt. Soc. Am. A 31(12), 2703-2710 (2014).

38. T. Ushiki, "Collagen fibers, reticular fibers and elastic fibers. A comprehensive understanding from a morphological viewpoint," Arch. Histol. Cytol. 65(2), 109-126 (2002).

39. L. Su, J. E. Siegel, and M. C. Fishbein, "Adipose tissue in myocardial infarction," Cardiovasc. Pathol. 13(2), 98-102 (2004).

40. M. F. G. Wood et al., "Polarization birefringence measurements for characterizing the myocardium, including healthy, infarcted, and stem-cell-regenerated tissues," J. Biomed. Opt. 15(4), 047009 (2010).

41. M. Mujat et al., "Autocalibration of spectral-domain optical coherence tomography spectrometers for in vivo quantitative retinal nerve fiber layer birefringence determination," J. Biomed. Opt. 12(4), 041205 (2007).

42. W. Yuan et al., "Optimal operational conditions for supercontinuumbased ultrahigh-resolution endoscopic OCT imaging," Opt. Lett. 41(2), 250-253 (2016).

43. Y. Gan et al., "Automated classification of optical coherence tomography images for human atrial tissue," J. Biomed. Opt. 21(10), 101407 (2015).

44. G. M. Fomovsky, S. Thomopoulos, and J. W. Holmes, "Contribution of extracellular matrix to the mechanical properties of the heart," J. Mol. Cell. Cardiol. 48(3), 490-496 (2010).

45. K. M. Myers et al., "A continuous fiber distribution material model for human cervical tissue," J. Biomech. 48(9), 1533-1540 (2015).

46. M.-R. Tsai et al., "Second-harmonic generation imaging of collagen fibers in myocardium for atrial fibrillation diagnosis," J. Biomed. Opt. 15(2), 026002 (2010).

47. A. Srinivasan and P. K. Sehgal, "Characterization of biocompatible collagen fibers-a promising candidate for cardiac patch," Tissue Eng. Part C: Methods 16(5), 895-903 (2009).

48. P. Whittaker et al., "Quantitative assessment of myocardial collagen with picrosirius red staining and circularly polarized light," Basic Res Cardiol. 89(5), 397-410 (1994).

Xinwen Yao received her MS in electrical engineering from Columbia University and BEng in measurement and control techology from Xiamen University. She is a doctoral candidate in electrical engineering at Columbia University.

Yu Gan received his MS in communications and information systems and electrical engineering Chinese Academy of Sciences and Stevens Institute of Technology, respectively, and his BS in electronic and information engineering from Nanjing University of Science and Technology. He is a doctoral candidate in electrical engineering at Columbia University.

Charles C. Marboe, MD, is professor of pathology and cell biology at Columbia University Medical Center. He has 34 years of experience in cardiovascular pathology.

Christine P. Hendon received her PhD in biomedical engineering from Case Western Reserve University and her BS in electrical engineering and computer science from the Massachusetts Institute of Technology. Her research interests are in developing biomedical optical systems and image analysis for applications for guidance of therapy. She is currently an assistant professor of electrical engineering at Columbia University. 